\title{
Measure Differential Inclusions: Existence Results and Minimum Problems
}

\author{
Luisa Di Piazza ${ }^{1}$ - Valeria Marraffa ${ }^{1}$ (D) . Bianca Satco ${ }^{2}$
}

Received: 30 December 2019 / Accepted: 27 September 2020 / Published online: 10 October 2020

(C) The Author(s) 2020

\begin{abstract}
We focus on a very general problem in the theory of dynamic systems, namely that of studying measure differential inclusions with varying measures. The multifunction on the right hand side has compact non-necessarily convex values in a real Euclidean space and satisfies bounded variation hypotheses with respect to the Pompeiu excess (and not to the HausdorffPompeiu distance, as usually in literature). This is possible due to the use of interesting selection principles for excess bounded variation set-valued mappings. Conditions for the minimization of a generic functional with respect to a family of measures generated by equiregulated left-continuous, nondecreasing functions and to associated solutions of the differential inclusion driven by these measures are deduced, under constraints only on the initial point of the trajectory.
\end{abstract}

Keywords Measure differential inclusion - Bounded variation · Pompeiu excess · Selection $\cdot$ Minimality condition

Mathematics Subject Classification (2010) 26A45 - 34A60 - 28B20 · 26A42 · 49Kxx

Valeria Marraffa

valeria.marraffa@unipa.it

Luisa Di Piazza

luisa.dipiazza@unipa.it

Bianca Satco

bianca.satco@eed.usv.ro

1 Department of Mathematics and Computer Science, University of Palermo, Via Archirafi 34, 90123, Palermo, Italy

2 Faculty of Electrical Engineering and Computer Science; Integrated Center for Research, Development and Innovation in Advanced Materials, Nanotechnologies, and Distributed Systems for Fabrication and Control (MANSiD), Stefan cel Mare University of Suceava, Universitatii 13, Suceava, Romania 


\section{Introduction}

In the dynamic of many systems in physics, engineering, biology or chemistry, one has to face the occurrence of discontinuities in the state, which can be seen as impulses. One way to mathematically describe such systems is offered by the theory of measure differential equations. On the other hand, in various situations (e.g. when a control is involved), it is more convenient to consider multivalued functions, i.e. differential inclusions driven by Borel measures (thus allowing a unified approach of differential or difference set-valued problems, of impulsive problems or even of dynamic inclusions on time scales $[11,19]$ ).

Usually in the literature concerning the theory of differential inclusions, the HausdorffPompeiu distance appears when writing the conditions imposed on the right-hand side. Relaxing the traditional hypotheses by using the Pompeiu excess (from the right or from the left) instead of Hausdorff-Pompeiu metric would be a consistent improvement.

In the present work, we study non-convex measure differential inclusions

$$
\begin{aligned}
& d x(t) \in G(t, x(t)) d \mu_{g}(t), \\
& x(0)=x_{0}
\end{aligned}
$$

with $x_{0} \in \mathbb{R}^{d}$, under excess bounded variation assumptions (inspired from [13], see also [12]) on the velocity set $G(t, x(t))$ and make use of interesting selection principles provided in this framework by V.V. Chistyakov and D. Repovš [13].

The map $G:[0,1] \times \mathbb{R}^{d} \rightarrow \mathcal{P}_{k}\left(\mathbb{R}^{d}\right)$ has compact possibly non-convex values and $g:[0,1] \rightarrow \mathbb{R}$ is a left-continuous nondecreasing function whose distributional derivative (i.e. the Stieltjes measure generated by $g$ ) is denoted by $\mu_{g}$.

Let us remark that it is unnatural to expect the solutions to be absolutely continuous or even continuous, and so, the considered space in which the theory is developed is the space of functions of bounded variation.

In Theorem 3 we obtain the existence of solutions defined by integrating in Stieltjes sense selections of bounded variation which, moreover, have some equiregulatedness property (by means of [20]). An instance where the hypotheses can be easily verified (Corollary 1), together with an example highlighting the generality of our assumptions are included.

Moreover, in Theorem 4 we get a compactness feature of the solution set of such a family of inclusions with varying measures, driven by an equiregulated sequence of functions of bounded variation.

As an application of the compactness result, we deduce (Theorem 6) a condition for minimizing a functional

$$
\int_{0}^{1} k\left(t, x(t), \mu_{g}\right) d t+l(x)
$$

over all $\mu_{g}$ in a set $\mathcal{G}$ of positive Borel measures on $[0,1]$ for which the inclusion (1.1) has solutions, and over all solutions $x$ driven by $\mu_{g}$. The functions $k:[0,1] \times \mathbb{R}^{d} \times \mathcal{G} \rightarrow \mathbb{R}_{+}$and $l: B V\left([0,1], \mathbb{R}^{d}\right) \rightarrow \mathbb{R}$, occurring in the minimization problem, satisfy lower semicontinuity assumptions.

This is an extension of a classical optimal control problem (which cannot be solved in the class of absolutely continuous functions for solutions, respectively of $L^{1}$ controls) to the space of functions of bounded variation for solutions, respectively of measures for controls (see $[29,31,32]$ treating the single-valued case or [23, 28]). For optimality conditions in the set-valued setting, but concerning different optimal control problems, we can refer to [39] (with convex velocity sets) or [14]. 
The novelty of our result comes from the completely different approach, based on compactness properties in the space of regulated functions [20] and on selection results for nonconvex-valued multifunctions of excess-bounded variation [13], this allowing to get minimality under new conditions comparing to the above mentioned works.

\section{Notions and Preliminary Facts}

In a traditional manner, for a function $u:[0,1] \rightarrow \mathbb{R}^{d}$ with values in a finite dimensional Banach space $\left(\mathbb{R}^{d},\|\|\right)$, the total variation will be denoted by

$$
\begin{aligned}
& \operatorname{var}(u,[0,1])= \\
& \sup \left\{\sum_{i=1}^{p}\left\|u\left(t_{i}\right)-u\left(t_{i-1}\right)\right\| ; \pi=\left\{t_{i}\right\}_{i=0}^{p} \text { finite partition of }[0,1], p \in \mathbb{N}\right\}
\end{aligned}
$$

and if it is finite then $u$ will be said to have bounded variation (or to be a BV function); $B V\left([0,1], \mathbb{R}^{d}\right)$ stands for the space of functions of bounded variation. The BV-norm is known as

$$
\|u\|_{B V}=\|u(0)\|+\operatorname{var}(u,[0,1]) .
$$

We endow this space with the two-norm topology; we recall that a sequence $\left(u_{n}\right)_{n} \subset$ $B V\left([0,1], \mathbb{R}^{d}\right)$ converges to $u$ in this topology if and only if

$$
u_{n} \rightarrow u \text { uniformly on }[0,1] \text { and }\left(\left\|u_{n}\right\|_{B V}\right)_{n} \text { is bounded. }
$$

As shown by Wiweger ([45]), there is a locally convex linear topology on $B V\left([0,1], \mathbb{R}^{d}\right)$, intermediate in strength to the topologies generated by the sup-norm and the $\|\cdot\|_{B V}$-norm, for which the convergence of a sequence coincides with the convergence in the two-norm sense.

Helly's selection principle will be an important tool in our work.

Theorem 1 Let $\left(u_{n}\right)_{n}$ be a sequence of functions defined on $[0,1]$ with values in $\mathbb{R}^{d}$. Suppose that it is uniformly bounded in variation by a constant $M$ and that the sequence $\left(u_{n}(0)\right)_{n}$ is bounded. Then there exists a subsequence pointwise convergent to a function $u$ with $\operatorname{var}(u,[0,1]) \leq M$.

We note (e.g. [4]) that if the functions take values in an infinite dimensional Banach space, then the boundedness condition on $\left(u_{n}(0)\right)_{n}$ is not enough to ensure the existence of a pointwise convergent subsequence; it is necessary to impose the relative compactness of $\left(u_{n}(t)\right)_{n}$ at any point $t \in[0,1]$.

For a real-valued $B V$-function $g$, by $\mu_{g}$ we denote its distributional derivative, which is in fact the corresponding Stieltjes measure. It is defined for half-open sub-intervals of $[0,1]$ by

$$
\mu_{g}([a, b))=g(b)-g(a)
$$

and it is then extended to all Borel subsets of the unit interval in the standard way.

Through the whole paper, for simplicity, we shall consider Stieltjes measures on $[0,1]$ generated by left-continuous nondecreasing functions which are null at 0 (without losing the generality, see [15, Remark 2.1]).

Classically (see [5]), a sequence $\left(\mu_{g_{n}}\right)_{n}$ of measures is said to be weakly* convergent to $\mu_{g}$ if $\int_{[0,1]} f(t) d \mu_{g_{n}}(t) \rightarrow \int_{[0,1]} f(t) d \mu_{g}(t)$ for every continuous function $f:[0,1] \rightarrow \mathbb{R}$, where the integrals are the Lebesgue-Stieltjes ones. 
Traditionally (cf. also [20]), a function $u:[0,1] \rightarrow \mathbb{R}^{d}$ is regulated if there exist the right and left limits $u(t+)$ and $u(s-)$ for every points $t \in[0,1)$ and $s \in(0,1]$ (the convention $u(0-)=u(0), u(1+)=u(1)$ is used). The set of discontinuity points of a regulated function is known to be at most countable ([33]) and the bounded variation functions (and also the continuous functions) are obviously regulated. Such functions are also bounded and the space of regulated functions is a Banach space when endowed with the sup-norm $\|\cdot\|_{C}$.

There is a notion strongly related to compactness properties in the space of regulated maps, that of equiregulatedness.

Definition 1 ([20]) A family $\mathcal{A}$ of regulated functions on $[0,1]$ with values in $\mathbb{R}^{d}$ is called equiregulated if for every $t_{0} \in[0,1]$ and every $\varepsilon>0$ one can find $\delta>0$ such that for any $u \in \mathcal{A}$

$$
\left\|u(t)-u\left(t_{0}-\right)\right\|<\varepsilon, \text { for every } t \in\left(t_{0}-\delta, t_{0}\right)
$$

and

$$
\left\|u(t)-u\left(t_{0}+\right)\right\|<\varepsilon, \text { for every } t \in\left(t_{0}, t_{0}+\delta\right)
$$

It was proved that:

Lemma 1 ([20, Corollary 2.4]) A set of $\mathbb{R}^{d}$-valued regulated functions on $[0,1]$ is relatively compact in the topology of uniform convergence if and only if it is equiregulated and pointwise bounded.

The characterization of equiregulatedness, given in [20], will be useful later:

Proposition 1 Let $\mathcal{A}$ be a set of regulated functions on $[0,1]$ with values in $\mathbb{R}^{d}$. The following assertions are equivalent:

(i) $\mathcal{A}$ is equiregulated and poinwisely relatively compact;

(ii) $\mathcal{A}(0)$ is bounded and there is an increasing continuous function $\eta:[0, \infty) \rightarrow[0, \infty)$, $\eta(0)=0$ and an increasing function $v:[0,1] \rightarrow[0,1], v(0)=0, v(1)=1$ such that for every $0 \leq t_{1}<t_{2} \leq 1$,

$$
\left\|u\left(t_{2}\right)-u\left(t_{1}\right)\right\| \leq \eta\left(v\left(t_{2}\right)-v\left(t_{1}\right)\right)
$$

for all $u \in \mathcal{A}$.

In the whole paper, we deal with the Lebesgue-Stieltjes integral $\int_{[0, t)} f(s) d \mu_{g}(s)$ (see $[6,27]$ or [33]), except for Section 4, where the Kurzweil-Stieltjes integral also appears. For the Kurzweil-Stieltjes integral, we use the notation $\int_{0}^{t} f(s) d g(s)$.

Definition 2 ([24, 35-37] or [43]) A function $f:[0,1] \rightarrow \mathbb{R}^{d}$ is said to be KurzweilStieltjes integrable with respect to $g:[0,1] \rightarrow \mathbb{R}$ on $[0,1]$ (shortly, $K S$-integrable) if there exists $\int_{0}^{1} f(s) d g(s) \in \mathbb{R}^{d}$ such that, for every $\varepsilon>0$, there is a positive function $\delta_{\varepsilon}$ on $[0,1]$ with

$$
\left\|\sum_{i=1}^{p} f\left(\xi_{i}\right)\left(g\left(t_{i}\right)-g\left(t_{i-1}\right)\right)-\int_{0}^{1} f(s) d g(s)\right\|<\varepsilon
$$

for every $\delta_{\varepsilon}$-fine partition $\left\{\left(\left[t_{i-1}, t_{i}\right], \xi_{i}\right): i=1, \ldots, p\right\}$ of $[0,1]$.

A partition $\left\{\left(\left[t_{i-1}, t_{i}\right], \xi_{i}\right): i=1, \ldots, p\right\}$ is $\delta_{\varepsilon}$-fine if for all $i=1, \ldots, p,\left[t_{i-1}, t_{i}\right] \subset$ ]$\xi_{i}-\delta_{\varepsilon}\left(\xi_{i}\right), \xi_{i}+\delta_{\varepsilon}\left(\xi_{i}\right)$ [. The $K S$-integrability is preserved on all sub-intervals of [0,1]. 
It is known that regulated functions are $K S$-integrable with respect to bounded variation functions and also bounded variation functions are $K S$-integrable with respect to regulated functions (see [43]). The properties of the primitive contained in the proposition below are important in order to establish the properties of our solutions.

Proposition 2 ([43, Proposition 2.3.16]) Let $g:[0,1] \rightarrow \mathbb{R}$ and $f:[0,1] \rightarrow \mathbb{R}^{d}$ be $K S$-integrable w.r.t. $g$. If $g$ is regulated, then so is the primitive $h:[0,1] \rightarrow \mathbb{R}^{d}, h(t)=$ $\int_{0}^{t} f(s) d g(s)$ and for every $t \in[0,1]$,

$$
h(t+)-h(t)=f(t)[g(t+)-g(t)] \quad \text { and } \quad h(t)-h(t-)=f(t)[g(t)-g(t-)] .
$$

It follows that $h$ is left-continuous, respectively right-continuous at the points where $g$ is. Besides, when $g$ is of bounded variation and $f$ is bounded, $h$ is also of bounded variation.

The following estimates hold.

Proposition 3 i) ([37, Lemma 1.4.16]) Let $f:[0,1] \rightarrow \mathbb{R}^{d}$ be regulated and $g:[0,1] \rightarrow \mathbb{R}$ be a $B V$ function. Then

$$
\left\|\int_{0}^{1} f(t) d g(t)\right\| \leq\|f\|_{C} \cdot \operatorname{var}(g,[0,1]) .
$$

ii) ([43, Theorem 2.3.8]) Let $f:[0,1] \rightarrow \mathbb{R}^{d}$ be a $B V$ function and $g:[0,1] \rightarrow \mathbb{R}$ be regulated. Then

$$
\begin{aligned}
\left\|\int_{0}^{1} f(t) d g(t)\right\| & \leq[\|f(0)\|+\|f(1)\|+\operatorname{var}(f,[0,1])]\|g\|_{C} \\
& \leq 2\|f\|_{B V}\|g\|_{C} .
\end{aligned}
$$

Note that the Lebesgue-Stieltjes integrability of a function $f$ implies the Kurzweil-Stieltjes integrability, but the two integrals do not always have the same value. More precisely, in the framework of a left-continuous nondecreasing function $g$, as a consequence of [27, Theorem 6.11.3] (see also [33, Theorem 8.1]), if $t \in[0,1]$ then

$$
\int_{0}^{t} f(s) d g(s)=\int_{[0, t]} f(s) d \mu_{g}(s)-f(t)(g(t+)-g(t))=\int_{[0, t)} f(s) d \mu_{g}(s) .
$$

Let us remind at this point that, by [3, Theorem 1], if a sequence $\left(g_{n}\right)_{n} \subset B V([0,1], \mathbb{R})$ two-norm converges to $g$, then the sequence of associated measures $\left(\mu_{g_{n}}\right)_{n}$ converges to $\mu_{g}$ in a sense which is stronger than the weak* convergence:

Theorem 2 ([3, Theorem 1]) Let $\left(g_{n}\right)_{n} \subset B V([0,1], \mathbb{R})$ two-norm converge to $g \in$ $B V([0,1], \mathbb{R})$. Then for each regulated $f:[0,1] \rightarrow \mathbb{R}$,

$$
\int_{0}^{1} f(s) d g_{n}(s) \rightarrow \int_{0}^{1} f(s) d g(s) .
$$

For all notions of set-valued analysis, we refer the reader to [2] or [8].

Let $\mathcal{P}_{k}\left(\mathbb{R}^{d}\right)$ be the space of all nonempty compact subsets of $\mathbb{R}^{d}$. It becomes a complete metric space when endowed with the Hausdorff-Pompeiu distance

$$
D\left(A, A^{\prime}\right)=\max \left(e\left(A, A^{\prime}\right), e\left(A^{\prime}, A\right)\right),
$$


where the (Pompeiu-) excess of the set $A \in \mathcal{P}_{k}\left(\mathbb{R}^{d}\right)$ over the set $A^{\prime} \in \mathcal{P}_{k}\left(\mathbb{R}^{d}\right)$ is defined by

$$
e\left(A, A^{\prime}\right)=\sup _{a \in A} \inf _{a^{\prime} \in A^{\prime}}\left\|a-a^{\prime}\right\| .
$$

A multifunction $\Gamma: \mathbb{R}^{d} \rightarrow \mathcal{P}_{k}\left(\mathbb{R}^{d}\right)$ is upper semicontinuous at a point $x_{0}$ if for every $\varepsilon>0$ there exists $\eta_{\varepsilon, x_{0}}>0$ such that $e\left(\Gamma(x), \Gamma\left(x_{0}\right)\right)<\varepsilon$ whenever $\left\|x-x_{0}\right\|<\eta_{\varepsilon, x_{0}}$, i.e.: $\Gamma(x) \subset \Gamma\left(x_{0}\right)+\varepsilon B$, where $B$ is the closed unit ball of $\mathbb{R}^{d}$.

For $A \in \mathcal{P}_{k}\left(\mathbb{R}^{d}\right)$, denote by $|A|=D(A,\{0\})$.

One says (see [13]) that a multifunction $F:[0,1] \rightarrow \mathcal{P}_{k}\left(\mathbb{R}^{d}\right)$ has bounded variation with respect to (shortly, w.r.t.) the excess function (at the right) or that it is of excess-bounded variation if

$$
\begin{aligned}
& V_{+}(F,[0,1])= \\
& \quad \sup \left\{\sum_{i=1}^{p} e\left(F\left(t_{i-1}\right), F\left(t_{i}\right)\right) ; \pi=\left\{t_{i}\right\}_{i=0}^{p} \text { partition of }[0,1], p \in \mathbb{N}\right\}<\infty .
\end{aligned}
$$

Similarly, one could define the excess variation at the left

$$
\begin{aligned}
& V_{-}(F,[0,1])= \\
& \quad \sup \left\{\sum_{i=1}^{p} e\left(F\left(t_{i}\right), F\left(t_{i-1}\right)\right) ; \pi=\left\{t_{i}\right\}_{i=0}^{p} \text { partition of }[0,1], p \in \mathbb{N}\right\}
\end{aligned}
$$

and if both $V_{+}(F,[0,1])$ and $V_{-}(F,[0,1])$ are finite, then $F$ has bounded variation in the sense of Hausdorff-Pompeiu distance (see [4]). Example 3.1 in [13] shows that, in an infinite dimensional Banach space, the condition to be of excess-bounded variation is strictly weaker than to be of bounded variation w.r.t. the Hausdorff-Pompeiu distance, which was traditionally used while studying measure differential inclusions (e.g. in $[17,18,34]$ or [38]).

As in [13], $F:[0,1] \rightarrow \mathcal{P}_{k}\left(\mathbb{R}^{d}\right)$ is called excess-Lipschitz continuous to the right with $L \geq 0$ if

$$
e(F(s), F(t)) \leq L(t-s), \text { whenever } 0 \leq s \leq t \leq 1
$$

and the infimum of such numbers $\mathrm{L}$ is called the excess-Lipschitz constant of the multifunction.

Let us finally recall ([13], page 884$)$ that $F:[0,1] \rightarrow \mathcal{P}_{k}\left(\mathbb{R}^{d}\right)$ is said to be excesscontinuous to the right (shortly, $C_{+}$) if

$$
\begin{aligned}
& \lim _{s \rightarrow t-} e(F(s), F(t))=0 \quad \text { for all } t \in(0,1] \\
& \text { and } \\
& \lim _{s \rightarrow t+} e(F(t), F(s))=0 \quad \text { for all } t \in[0,1) \text {. }
\end{aligned}
$$

\section{Existence Results for Measure Differential Inclusions}

First of all, we notice that when the measure driving the problem is a Stieltjes measure generated by an absolutely continuous function, then by solution we mean solution in the classical (Carathéodory) sense which, obviously, coincides with those defined below. In the more general case, where the measure is generated by a nondecreasing function, in literature there are various ways to choose the solution concept of a measure differential problem, see $[7,15,22,25,46]$ or [38]. For instance, in the single-valued case, in [31, Definition 3.1] the 
following notion of generalized solution, in [46, Definition 5.3.2] the notion of $V$-solution and in [1] the notion of simple limit solution have been given.

Definition 3 i) A pair $(x, g)$ is a generalized solution of (1.1) if there exists a sequence $\left(x_{n}, g_{n}\right)_{n} \in W^{1,1}\left([0,1], \mathbb{R}^{d}\right) \times W^{1,1}([0,1], \mathbb{R})$ satisfying the inclusion such that $\left(x_{n}\right)_{n}$ converges to $x$ in the sense that

$$
x_{n}(0) \rightarrow x(0) \text { and } d x_{n} \rightarrow d x \text { weakly* }
$$

and $\left(g_{n}\right)_{n}$ converges to $g$ in the sense that

$$
g_{n}(0) \rightarrow g(0) \text { and } d g_{n} \rightarrow d g \text { weakly* }
$$

ii) A function $x:[0,1] \rightarrow \mathbb{R}^{d}$ is a $V$-solution of (1.1) for some nondecreasing function $V:[0,1] \rightarrow \mathbb{R}$ if there exists a sequence of absolutely continuous functions $v_{k}:[0,1] \rightarrow \mathbb{R}$ such that for each $t \in v_{k}[0,1]$,

$$
v_{k}(t) \rightarrow g(t) \text { and } \operatorname{var}\left(v_{k},[0, t]\right) \rightarrow V(t)
$$

and there exists a sequence of solutions $x_{k}$ of problem (1.1) driven by $\mu_{v_{k}}$ pointwise convergent to $x$.

iii) A function $x:[0,1] \rightarrow \mathbb{R}^{d}$ is a BV simple limit solution of (1.1) if there exists a sequence of absolutely continuous functions $v_{k}:[0,1] \rightarrow \mathbb{R}$ with equibounded variation such that for each $t \in[0,1]$,

$$
v_{k}(t) \rightarrow g(t) \quad \text { and } \quad\left\|v_{k}-g\right\|_{L^{1}} \rightarrow 0
$$

and there exists a sequence of solutions $x_{k}$ of problem (1.1) driven by $\mu_{v_{k}}$ satisfying

$$
x_{k}(t) \rightarrow x(t) \text { and }\left\|x_{k}-x\right\|_{L^{1}} \rightarrow 0 .
$$

Note that the concept of $B V$ simple limit solution recalled from [1] has been extended in [28] to the case of the problem

$$
\begin{array}{r}
x^{\prime}(t)=g(x(t), u(t), v(t)) \cdot u^{\prime}(t) \quad \text { a.e.t } \in[0,1] \\
x(0)=x_{0}, u(0)=u_{0}
\end{array}
$$

which, when $v(t) \in V$, where $V$ is a compact subset of $\mathbb{R}^{d}$, becomes a set-valued control problem of type (1.1).

The generalized solution given in $i$ ) ([31]) is proved to be equivalent to a notion of solution defined through an extended graph completion, related to those defined via graph completion methods in other works, such as [15] or [38]. Likewise, the notion of BV-simple limit solution been shown in [1, Theorem 4.2] to be equivalent to that of graph completion solution (see [31, Section 3] or [1] for a more extensive discussion). For alternative type of solution, via a notion of extended trajectory, for the inclusion (3.1) involved in solving practical optimal control problems we can also refer the reader to [23].

Let us specify the notion of solution considered in this paper (we shall see later, in Proposition 4, connections with the above mentioned kinds of solutions).

Definition 4 ([9]) A solution of the problem (1.1) is a function $x:[0,1] \rightarrow \mathbb{R}^{d}$ for which there exists $f:[0,1] \rightarrow \mathbb{R}^{d}$ Lebesgue-Stieltjes integrable w.r.t. $\mu_{g}$ such that $f(t) \in G(t, x(t-)) \mu_{g}$-a.e. and

$$
x(t)=x_{0}+\int_{[0, t)} f(s) d \mu_{g}(s), \forall t \in[0,1] .
$$


By basic properties of Lebesgue-Stieltjes integrals, $x \in B V\left([0,1], \mathbb{R}^{d}\right)$ and leftcontinuous (since $g$ is left-continuous, by Proposition 2) and so, in the preceding definition, we may write $f(t) \in G(t, x(t)) \mu_{g}$-a.e.

This definition has the advantages to be the most natural generalization from the classical case (where the solutions can be put in integral form) and to be easy to handle, through the theory of Stieltjes integrals.

In the case of a finite dimensional space, existence results for this kind of solution can be found for single-valued framework in $[19,26]$ or [21], while for the set-valued setting, when the multifunction $G$ has compact convex values, existence results were proved in [9] under Carathéodory-type assumptions or in [17]. Moreover, well-posedness has been obtained (e.g. [40]) and continuous dependence results were given (in [34] under bounded variation assumptions w.r.t. the Hausdorff-Pompeiu distance, in [10] when the sequence of measures was supposed to converge in some sense strictly related to the set-valued setting, or in [18] using the notion of uniformly bounded $\varepsilon$-variation introduced in [20]).

Now, we present an existence result for non-convex differential inclusions driven by measures by imposing only conditions involving the Pompeiu excess (instead of HausdorffPompeiu distance, as in previously mentioned works).

We shall see later that the family of such solutions (with specific properties, namely given by equiregulated BV selections) possesses interesting compactness properties.

Before proceeding with the very first step, the existence theorem, we stress that an important tool in our study consists in using appropriate selections results. In [13] one can find several interesting results in this direction.

Thus, [13, Theorem 1] states that a multifunction of excess-bounded variation possesses bounded variation selections with total variation smaller than the excess variation of the multifunction.

Notably, [13, Theorem 3] asserts that if moreover the set-valued map is of excess bounded variation to the right and excess continuous to the right, then it possesses a continuous selection of bounded variation (with the same property, that its total variation is majorized by the excess variation of the multifunction).

In fact, taking a look in the proof of [13, Theorem 3], we may see that a deeper result is hidden therein, and this will be a key point in our proofs.

Lemma 2 Let $F:[0,1] \rightarrow \mathcal{P}_{k}\left(\mathbb{R}^{d}\right)$ be of excess-bounded variation. For each $t \in[0,1]$, denote by $v(t)=V_{+}(F,[0, t])$. Then there exists a selection $f$ of $F$ such that

$$
\|f(t)-f(s)\| \leq v(t)-v(s), \forall 0 \leq s \leq t \leq 1 .
$$

Remark 1 It implies that $\operatorname{var}(f,[0,1]) \leq V_{+}(F,[0,1])$ and not only on the unit interval, but also on any subinterval of $[0,1]$

Proof Let $l=V_{+}(F,[0,1])$. Then $v([0,1]) \subset[0, l]$. Obviously, $v$ is nondecreasing, thus regulated but not necessarily continuous.

Take $s \in v([0,1])$ and consider $v^{-1}(s)=\{t \in[0,1] ; v(t)=s\}$ which is a nonempty subset of the unit interval.

Define the nonempty compact-valued multifunction $G: v([0,1]) \rightarrow \mathcal{P}_{k}\left(\mathbb{R}^{d}\right)$ by

$$
G(s)=\bigcap_{t \in v^{-1}(s)} F(t) .
$$

We shall prove that $G$ is excess-Lipschitz continuous, with Lipschitz constant $\leq 1$. 
Let $s_{1}<s_{2} \in v([0,1])$. Then for each $t \in v^{-1}\left(s_{1}\right)$,

$$
e\left(G\left(s_{1}\right), G\left(s_{2}\right)\right) \leq e\left(F(t), G\left(s_{2}\right)\right) .
$$

As shown in [13], for any $0 \leq t^{\prime} \leq t^{\prime \prime} \leq 1, t^{\prime}, t^{\prime \prime} \in v^{-1}\left(s_{2}\right)$ one has

$$
F\left(t^{\prime}\right) \subseteq F\left(t^{\prime \prime}\right)
$$

so, taking into account that any decreasing sequence of compact sets converges in Hausdorff-Pompeiu distance to their intersection (see e.g. [42], page 7), one can deduce that for an arbitrary $\varepsilon>0$ we are able to choose $t^{\prime} \in v^{-1}\left(s_{2}\right)$ such that

$$
e\left(F\left(t^{\prime}\right), G\left(s_{2}\right)\right) \leq \varepsilon .
$$

It follows that

$$
\begin{aligned}
e\left(G\left(s_{1}\right), G\left(s_{2}\right)\right) & \leq e\left(F(t), G\left(s_{2}\right)\right) \\
& \leq e\left(F(t), F\left(t^{\prime}\right)\right)+e\left(F\left(t^{\prime}\right), G\left(s_{2}\right)\right) \\
& \leq e\left(F(t), F\left(t^{\prime}\right)\right)+\varepsilon \\
& \leq v\left(t^{\prime}\right)-v(t)+\varepsilon .
\end{aligned}
$$

But $t \in v^{-1}\left(s_{1}\right)$ and $t^{\prime} \in v^{-1}\left(s_{2}\right)$, therefore

$$
v(t)=s_{1} \quad \text { and } \quad v\left(t^{\prime}\right)=s_{2} .
$$

As $\varepsilon$ was arbitrarily chosen, one gets

$$
e\left(G\left(s_{1}\right), G\left(s_{2}\right)\right) \leq s_{2}-s_{1}
$$

and the excess-Lipschitz continuity of $G$ is proved.

Then, by a remark made by the authors themselves at page 875 , [13, Theorem 2] holds more generally on a nonempty set of real numbers (not necessarily interval), and applying it one gets that $G$ has a Lipschitz-continuous selection $g$ with Lipschitz constant 1 . Then $f=g \circ v$ is BV, it is a selection of $F$ and, besides, for any $0 \leq s \leq t \leq 1$,

$$
\|f(t)-f(s)\| \leq v(t)-v(s) .
$$

The existence result reads as follows.

Theorem 3 Let $G:[0,1] \times \mathbb{R}^{d} \rightarrow \mathcal{P}_{k}\left(\mathbb{R}^{d}\right)$ satisfy the hypotheses below:

1) For each $t \in[0,1], G(t, \cdot)$ is upper semicontinuous;

2) For every $B V$-function $x:[0,1] \rightarrow \mathbb{R}^{d}$, the map $G(\cdot, x(\cdot))$ has excess-bounded variation;

3) One can find $R_{0}>0$ and $M_{R_{0}}>0$ such that for every left-continuous $B V$-function $x:[0,1] \rightarrow \mathbb{R}^{d}$ and every left-continuous nondecreasing function $h:[0,1] \rightarrow \mathbb{R}$ such that

$$
\operatorname{var}(x,[s, t)) \leq R_{0} \cdot(h(t)-h(s)) \text { for all } 0 \leq s<t \leq 1
$$

one has:

$$
V_{+}(G(\cdot, x(\cdot)),[s, t)) \leq M_{R_{0}} \cdot(h(t)-h(s)), \text { for all } 0 \leq s<t \leq 1 .
$$

Let $g:[0,1] \rightarrow \mathbb{R}$ be a left-continuous nondecreasing function satisfying the inequality

$$
g(1)-g(0) \leq \frac{R_{0}-\left|G\left(0, x_{0}\right)\right|}{M_{R_{0}}} .
$$


Then there exists at least one solution for the measure differential problem (1.1) driven by $\mu_{g}$ such that, for all $t \in[0,1], x(t)=x_{0}+\int_{[0, t)} f(s) d \mu_{g}(s)$, where $f$ verifies $f(t) \in$ $G(t, x(t))$ for all $t \in[0,1]$ and

$$
\|f(t)-f(s)\| \leq M_{R_{0}} \cdot(g(t)-g(s)) \text { for every } 0 \leq s<t \leq 1 .
$$

Remark 2 As a consequence, $f$ is $B V$ and $\operatorname{var}(f,[0,1]) \leq M_{R_{0}} \cdot(g(1)-g(0))$, and by Proposition 2 also the solution $x$ is $B V$.

Moreover, we can see from the fact that the existing solution is obtained via a selection $f$ with

$$
\|f(t)-f(s)\| \leq M_{R_{0}} \cdot(g(t)-g(s)) \text { for every } 0 \leq s<t \leq 1
$$

that when $g$ is continuous (such as, in a case of an absolutely continuous function $g$ ), the selection $f$ is also continuous.

Proof The idea is to construct a sequence of approximate solutions (which are $B V$ equiregulated functions) and to show that it is possible to extract a convergent subsequence by Helly's selection principle.

Thus, let $x_{0}(t)=x_{0}$ for $t \in[0,1]$. Suppose that we have already constructed a $B V$ function $x_{n}$ on $[0,1]$ with $\operatorname{var}\left(x_{n},[s, t)\right) \leq R_{0} \cdot(g(t)-g(s))$ for all $0 \leq s<t \leq 1$ and choose $x_{n+1}$ by the following steps.

Using hypothesis 2), we apply Lemma 2. By 3)

$$
V_{+}\left(G\left(\cdot, x_{n}(\cdot)\right),[s, t)\right) \leq M_{R_{0}} \cdot(g(t)-g(s)) \forall 0 \leq s<t \leq 1
$$

and we obtain the existence of a selection $f_{n}(t) \in G\left(t, x_{n}(t)\right), \forall t \in[0,1]$ with

$$
\left\|f_{n}(t)-f_{n}(s)\right\| \leq M_{R_{0}} \cdot(g(t)-g(s)) \forall 0 \leq s<t \leq 1
$$

(it will follow that $\left.\operatorname{var}\left(f_{n},[0,1]\right) \leq M_{R_{0}} \cdot(g(1)-g(0))\right)$.

Define now

$$
x_{n+1}(t)=x_{0}+\int_{[0, t)} f_{n}(s) d \mu_{g}(s), \quad \forall t \in[0,1] .
$$

Since $f_{n}$ is bounded and taking into account equality (2.1), also $x_{n+1}$ is of bounded variation by Proposition 2.

We can see, by [27, Theorem 6.3.4], that for any $s<t$,

$$
\begin{aligned}
\operatorname{var}\left(x_{n+1},[s, t)\right) & \leq\left(\left\|f_{n}(0)\right\|+\operatorname{var}\left(f_{n},[0,1]\right)\right) \cdot(g(t)-g(s)) \\
& \leq\left(\left|G\left(0, x_{0}\right)\right|+M_{R_{0}}(g(1)-g(0)) \cdot(g(t)-g(s))\right. \\
& \leq R_{0} \cdot(g(t)-g(s)),
\end{aligned}
$$

and so, by (3.3), the process can be continued.

Now, the sequence $\left(f_{n}\right)_{n}$ is bounded in variation and $f_{n}(0) \in G\left(0, x_{0}\right)$. Thus, by Helly's selection principle, one can extract a subsequence $\left(f_{n_{k}}\right)_{k}$ pointwise convergent to a $B V$ function $f$.

Since by Proposition 1 the sequence $\left(f_{n_{k}}\right)_{k}$ is also equiregulated, Lemma 1 implies that we can suppose that the subsequence is uniformly convergent to $f$, thus $f$ satisfies also the condition

$$
\|f(t)-f(s)\| \leq M_{R_{0}} \cdot(g(t)-g(s)) \text { for every } 0 \leq s<t \leq 1 .
$$

Next, since $\left(f_{n_{k}}\right)_{k}$ converges uniformly to $f$, it follows (Proposition 3 ) that

$$
\int_{[0, t)} f_{n_{k}}(s) d \mu_{g}(s) \rightarrow \int_{[0, t)} f(s) d \mu_{g}(s)
$$


and so, if we denote by

$$
x(t)=x_{0}+\int_{[0, t)} f(s) d \mu_{g}(s),
$$

obviously $x_{n_{k}} \rightarrow x$ pointwise.

We assert that $x$ is a solution for our measure driven differential inclusion (i.e, $f(t) \in$ $G(t, x(t)) \mu_{g}$-a.e.). Using hypothesis 1$)$, for each $t \in[0,1]$ and $\varepsilon>0$, the exists $k_{\varepsilon, t} \in \mathbb{N}$ such that

$$
G\left(t, x_{n_{k}}(t)\right) \subset G(t, x(t))+\varepsilon B
$$

for all $k$ greater than $k_{\varepsilon, t}$, therefore (since $G(t, x(t))+\varepsilon B$ is closed) $\left.f(t) \in G(t, x(t))\right)+\varepsilon B$, and then the arbitrariness of $\varepsilon$ implies $f(t) \in G(t, x(t))$.

Remark 3 We observe that in Theorem 3 it suffices to assume that the condition (3.2) holds only for the left-continuous nondecreasing function $g$ related to the differential inclusion (1.1) (and not for all $h$ with the announced property). However, in order to obtain continuous dependence on the measures driving the inclusion and relations with other types of solutions it is preferable to impose the more general assumption (3.2).

Proposition 4 If $G$ and $g$ satisfy the assumptions of Theorem 3 and $g$ is continuous, then:

i) there are generalized solutions $(x, g)$ (by means of Definition 3.i)) of (1.1) such that $x$ is also a solution of (1.1);

ii) for a suitable $V$ there are $V$-solutions (by means of Definition 3.ii)) of (1.1) which are also solutions of (1.1);

iii) there are BV-simple limit solutions of (1.1) which are solutions of (1.1) as well.

Proof Let $\left(v_{k}\right)_{k}$ be a sequence of absolutely continuous, nondecreasing functions, uniformly convergent to $g$ (e.g. the Bernstein polynomials associated to $g$ [30, Theorem 7.1.2. and Theorem 7.1.5]).

Without loss of generality $v_{k}(0)=g(0)=0$ and $v_{k}(1)=g(1)$, so $\operatorname{var}\left(v_{k},[0,1]\right)=$ $v_{k}(1)-v_{k}(0)=\operatorname{var}(g,[0,1])=g(1)-g(0)$ for every $k \in \mathbb{N}$.

Our Theorem 3 applies to the measure differential inclusion (1.1) driven by the measure $\mu_{v_{k}}$ and then there exists a solution $y_{k}$ defined by

$$
y_{k}(t)=x_{0}+\int_{[0, t)} f_{k}(s) d \mu_{v_{k}}(s), \forall t \in[0,1]
$$

through a selection $f_{k}$ of $G\left(\cdot, y_{k}(\cdot)\right)$ satisfying the condition

$$
\left\|f_{k}(t)-f_{k}(s)\right\| \leq M_{R_{0}} \cdot\left(v_{k}(t)-v_{k}(s)\right) \text { for every } 0 \leq s<t \leq 1,
$$

whence

$$
\operatorname{var}\left(f_{k},[0,1]\right) \leq M_{R_{0}} \cdot g(1) \text {. }
$$

So, $\left(f_{k}\right)_{k}$ is bounded in variation and, besides, $f_{k}(0) \in G\left(0, x_{0}\right), \forall k$. An application of Helly's selection theorem implies that it has a subsequence $\left(f_{n_{k}}\right)_{k}$ pointwise convergent to a $B V$ function $f$ with $\operatorname{var}(f) \leq M_{R_{0}} \cdot g(1)$.

Let

$$
x(t)=x_{0}+\int_{[0, t)} f(s) d \mu_{g}(s), \quad \forall t \in[0,1] .
$$

As $\left(v_{n_{k}}\right)_{k}$ uniformly converges to $g$ and $\left(f_{n_{k}}\right)_{k}$ is bounded in variation, by Proposition 3 , for each $t \in[0,1]$,

$$
\int_{[0, t)} f_{n_{k}}(s) d \mu_{v_{n_{k}}}(s)-\int_{[0, t)} f_{n_{k}}(s) d \mu_{g}(s) \rightarrow 0 .
$$


Moreover, as $\left(f_{n_{k}}\right)_{k}$ pointwise converges to $f$ and it is dominated by the constant (therefore, integrable) function $\left|G\left(0, x_{0}\right)\right|+M_{R_{0}} \cdot g(1)$,

Consequently,

$$
\int_{[0, t)} f_{n_{k}}(s) d \mu_{g}(s) \rightarrow \int_{[0, t)} f(s) d \mu_{g}(s) .
$$

$$
y_{n_{k}}(t)=x_{0}+\int_{[0, t)} f_{n_{k}}(s) d \mu_{v_{n_{k}}}(s) \rightarrow x_{0}+\int_{[0, t)} f(s) d \mu_{g}(s)=x(t)
$$

pointwise.

Finally, $x$ is a solution in the sense of Definition 4 of our problem since

$$
x(t)=x_{0}+\int_{[0, t)} f(s) d \mu_{g}(s), \quad \forall t \in[0,1],
$$

and by the upper semicontinuity condition imposed on $G, f(t) \in G(t, x(t))$ for every $t \in[0,1]$.

i) Clearly, $v_{k}$ and $y_{n_{k}}, k \in \mathbb{N}$ are absolutely continuous and $y_{n_{k}}(0)=x_{0} \rightarrow x(0)=x_{0}$, $v_{k}(0)=0 \rightarrow g(0)=0$.

Since

$$
\operatorname{var}\left(y_{n_{k}},[0,1]\right) \leq\left\|f_{n_{k}}\right\|_{C} \cdot \operatorname{var}\left(v_{n_{k}},[0,1]\right) \leq\left(\left|G\left(0, x_{0}\right)\right|+M_{R_{0}} \cdot g(1)\right) \cdot g(1)
$$

it follows, by Helly's convergence result, that $d y_{n_{k}} \rightarrow d x$ weakly* (by the same reason, $d v_{k} \rightarrow d g$ weakly*), thus $(x, g)$ is a generalized solution of (1.1).

ii) Denoting by $V(t)=g(t)-g(0)$, one can see that $\left(v_{k}\right)_{k}$ is $V$-convergent to $g$, i.e. it converges pointwise to $g$ and $\operatorname{var}\left(v_{k},[0, t]\right) \rightarrow V(t)$. As $y_{n_{k}}(t) \rightarrow x(t)$ pointwise, one concludes that $x$ is a $V$-solution of (1.1) as well.

iii) Obviously, the sequence $\left(v_{k}\right)_{k}$ has equibounded variation. Besides, by dominated convergence theorem, $\left\|v_{k}-g\right\|_{L^{1}} \rightarrow 0$ as $k \rightarrow \infty$.

Also,

$$
\left\|y_{n_{k}}(t)\right\| \leq\left(\left|G\left(0, x_{0}\right)\right|+M_{R_{0}} \cdot g(1)\right) \cdot \operatorname{var} v_{k} \leq\left(\left|G\left(0, x_{0}\right)\right|+M_{R_{0}} \cdot g(1)\right) \cdot g(1)
$$

and, as before, by dominated convergence result, $\left\|y_{n_{k}}-x\right\|_{L^{1}} \rightarrow 0$ as $k \rightarrow \infty$.

In the same line, of the connections between various types of solutions, we indicate the following example.

Example 1 Consider the problem

$$
d x(t)=x(t) d g(t), \quad x(0)=x_{0},
$$

where

$$
g(t)=\left\{\begin{array}{l}
0, t \in\left[0, \frac{1}{2}\right] \\
1, t \in\left(\frac{1}{2}, 1\right] .
\end{array}\right.
$$

Defining for each $k \in \mathbb{N}, k \geq 3$,

$$
v_{k}(t)=\left\{\begin{array}{l}
0, t \in\left[0, \frac{1}{2}\right] \\
k\left(t-\frac{1}{2}\right), t \in\left(\frac{1}{2}, \frac{1}{2}+\frac{1}{k}\right] \\
1, t \in\left(\frac{1}{2}+\frac{1}{k}, 1\right]
\end{array}\right.
$$

one gets an equibounded in variation sequence of absolutely continuous functions, convergent towards $g$ pointwise and also in $L^{1}$-norm. 
The sequence of solutions of our problem driven by $v_{k}$ given by

$$
x_{k}(t)=x_{0} \cdot e^{v_{k}(t)}, t \in[0,1]
$$

converges pointwise and (by dominated convergence theorem) in $L^{1}$-norm to the function defined on the unit interval by

$$
x(t)=x_{0} \cdot e^{g(t)}, t \in[0,1]
$$

which makes it a BV simple limit solution for our problem.

On the other hand, $x$ is not a solution in the sense of Definition 4 since

$$
x\left(\frac{1}{2}+\right)-x\left(\frac{1}{2}\right)=x_{0}(e-1)
$$

while

$$
\Delta^{+}\left(\int_{[0, t)} x(s) d g(s)\right)\left(\frac{1}{2}\right)=x\left(\frac{1}{2}\right) \cdot \Delta^{+} g\left(\frac{1}{2}\right)=x_{0},
$$

where if $l(t)$ is a function, $\Delta^{+} l(t)=l(t+)-l(t)$. To complete the discussion, we notice that the function

$$
x(t)=\left\{\begin{array}{l}
x_{0}, t \in\left[0, \frac{1}{2}\right] \\
2 x_{0}, t \in\left(\frac{1}{2}, 1\right]
\end{array}\right.
$$

is a solution in the sense of Definition 4 to this problem.

In view of the technical appearance of the hypothesis of Theorem 3, we think it is useful to present a consequence.

Corollary 1 Let $G:[0,1] \times \mathbb{R}^{d} \rightarrow \mathcal{P}_{k}\left(\mathbb{R}^{d}\right)$ verify hypothesis 1$)$ in Theorem 3. Suppose that there exists $L>0$ satisfying the following assumption:

$$
e\left(G\left(t_{1}, x\right), G\left(t_{2}, y\right)\right) \leq L\|x-y\|, \quad \text { for every } t_{1}<t_{2}, \quad \text { and for every } x, y \in \mathbb{R}^{d} .
$$

Then for any left-continuous nondecreasing function $g:[0,1] \rightarrow \mathbb{R}$ with

$$
L \cdot(g(1)-g(0))<1,
$$

there exists at least one solution for the measure differential problem (1.1) such that, for all $t \in[0,1], x(t)=x_{0}+\int_{[0, t)} f(s) d \mu_{g}(s)$, where $f$ verifies $f(t) \in G(t, x(t))$ for all $t \in[0,1]$ and

$$
\|f(t)-f(s)\| \leq \frac{L\left|G\left(0, x_{0}\right)\right|}{1-L(g(1)-g(0))} \cdot(g(t)-g(s)) \quad \text { for every } 0 \leq s<t \leq 1 .
$$

Proof Let us see that for any left-continuous $B V$-function $x:[0,1] \rightarrow \mathbb{R}^{d}$ and any $0 \leq$ $s<t \leq 1$,

$$
\begin{aligned}
V_{+}(G(\cdot, x(\cdot)),[s, t)) & =\sup _{\pi \text { partition of }[s, t)} \sum_{i=1}^{n} e\left(G\left(t_{i-1}, x\left(t_{i-1}\right)\right), G\left(t_{i}, x\left(t_{i}\right)\right)\right) \\
& \leq \sup _{\pi \operatorname{partition} \text { of }[s, t)} \sum_{i=1}^{n} L\left\|x\left(t_{i-1}\right)-x\left(t_{i}\right)\right\| \\
& \leq \operatorname{Lvar}(x,[s, t)),
\end{aligned}
$$

whence $G(\cdot, x(\cdot))$ is of excess-bounded variation. 
Moreover, if $x$ and $h$ are such that $\operatorname{var}(x,[s, t)) \leq R_{0} \cdot(h(t)-h(s))$ for any $0 \leq s<$ $t \leq 1$, then

$$
\left.V_{+}(G(\cdot, x(\cdot)),[s, t)) \leq L \cdot R_{0} \cdot(h(t)-h(s))\right), \forall 0 \leq s<t \leq 1
$$

and so, we can take $M_{R_{0}}=L \cdot R_{0}$ and hypothesis 3 ) is also satisfied by any $R_{0}$ and $M_{R_{0}}=L \cdot R_{0}$.

Now if $g:[0,1] \rightarrow \mathbb{R}$ is left-continuous nondecreasing with $L(g(1)-g(0))<1$, by choosing $R_{0}=\frac{\left|G\left(0, x_{0}\right)\right|}{1-L(g(1)-g(0))}$ we obtain that

$$
g(1)-g(0)=\frac{R_{0}-\left|G\left(0, x_{0}\right)\right|}{M_{R_{0}}}
$$

and so, we can apply Theorem 3 to get the existence of at least one solution for the measure differential problem (1.1) such that $x(t)=x_{0}+\int_{[0, t)} f(s) d \mu_{g}(s)$, for all $t \in[0,1]$ and $f(t) \in G(t, x(t))$ verifies for every $0 \leq s<t \leq 1$,

$$
\|f(t)-f(s)\| \leq M_{R_{0}} \cdot(g(t)-g(s))=\frac{L\left|G\left(0, x_{0}\right)\right|}{1-L(g(1)-g(0))} \cdot(g(t)-g(s)) .
$$

Remark 4 The assumption of the preceding Corollary is satisfied if

i) If $t_{1} \leq t_{2}$, then $G\left(t_{1}, x\right) \subset G\left(t_{2}, x\right)$ for every $x \in \mathbb{R}^{d}$;

ii) There exists $L>0$ such that $D(G(t, x), G(t, y)) \leq L\|x-y\|$ for every $t \in[0,1]$, $x, y \in \mathbb{R}^{d}$.

since for all $t_{1}<t_{2}$ and $x, y \in \mathbb{R}^{d}$,

$$
\begin{aligned}
e\left(G\left(t_{1}, x\right), G\left(t_{2}, y\right)\right) & \leq e\left(G\left(t_{1}, x\right), G\left(t_{1}, y\right)\right)+e\left(G\left(t_{1}, y\right), G\left(t_{2}, y\right)\right) \\
& \leq L\|x-y\|+0=L\|x-y\| .
\end{aligned}
$$

In [13] it can be found an interesting example showing that, in an infinite dimensional Banach space, the excess bounded variation condition is strictly weaker than the bounded variation w.r.t. the Hausdorff-Pompeiu metric. We shall see now a simple example highlighting the same feature in finite dimensional spaces.

Example 2 Let $\left(t_{n}\right)_{n} \subset[0,1]$ be an increasing sequence converging to 1 (where $t_{1}=0$ ) and let $\left(x_{n}\right)_{n}$ be a sequence on the unit circle $S=\left\{x \in \mathbb{R}^{2}:\|x\|=1\right\}$ defined as described in the sequel.

At the step 1 , consider the point $x_{1}=(1,0)$.

At the step 2, take the points which divide the circle into $2^{1}$ equal parts (starting with $x_{1}$ ) and eliminate the point considered at the step 1; denote this point by $x_{2}$.

Similarly, at the step $n+1(n \in \mathbb{N})$ take the points dividing the circle into $2^{n}$ equal parts (starting with $x_{1}$ ) and eliminate the points considered at the previous steps; denote the remaining points, ordered clockwise, by $x_{2^{n-1}+1}, \ldots, x_{2^{n}}$.

Remark that $\left\|x_{1}-x_{2}\right\|=2$ and that, for each regular polygon with $2^{n}$ edges inscribed in the unit circle, the length of its sides satisfies the inequality

$$
l_{2^{n}} \geq \frac{\sqrt{2}}{2^{n-2}} .
$$


Consider $F:[0,1] \rightarrow 2^{\mathbb{R}^{2}}$ the multifunction given by

$$
F(t)= \begin{cases}\left\{x_{k}\right\}_{k=1}^{n} & \text { if } t \in\left[t_{n}, t_{n+1}\right), n=1,2, \ldots ; \\ \partial B & \text { if } t=1 .\end{cases}
$$

First of all, we observe that the multifunction has compact (nonempty) values. Then, let us verify that $F$ has excess bounded variation.

For any $t^{\prime} \leq t^{\prime \prime} \in[0,1]$, it can be immediately seen that $F\left(t^{\prime}\right) \subseteq F\left(t^{\prime \prime}\right)$, therefore

$$
V_{+}(F,[0,1])=0 .
$$

Let us finally check that the total variation of $F$ w.r.t. the Hausdorff-Pompeiu distance is infinite. We may write, for every $n \in \mathbb{N}, n \geq 2$ :

$$
\begin{aligned}
V_{-}(F,[0,1]) & \geq \sum_{k=1}^{2^{n}-1} e\left(F\left(t_{k+1}\right), F\left(t_{k}\right)\right) \\
& =\sum_{k=1}^{2^{n}-1} \min _{i=1}^{k}\left\|x_{k+1}-x_{i}\right\| \\
& =\left\|x_{2}-x_{1}\right\|+\left\|x_{3}-x_{1}\right\|+\left\|x_{4}-x_{1}\right\|+\ldots \\
& =\left\|x_{2}-x_{1}\right\|+2 l_{2^{2}}+\ldots+2^{n-1} l_{2^{n}} \\
& \geq 2+2 \cdot \sqrt{2}+4 \cdot \frac{\sqrt{2}}{2}+\ldots+2^{n-1} \cdot \frac{\sqrt{2}}{2^{n-2}} \\
& \geq 2+2(n-1) \sqrt{2}
\end{aligned}
$$

which, obviously, tends to $\infty$ when $n \rightarrow \infty$. Thus, the multifunction has not bounded variation w.r.t. the Hausdorff-Pompeiu metric.

\section{Minimality Conditions}

\subsection{Compactness Properties of the Solution Set}

We study in this section compactness properties of the solutions set of (1.1), essential for getting the announced minimality result.

For each $n \in \mathbb{N}$, let $\mathcal{S}_{g_{n}}$ be the set of solutions for the problem (1.1) driven by the distributional derivative $\mu_{g_{n}}$ of a left-continuous nondecreasing function $g_{n}$, and let $f_{n}$ be the corresponding selection (see Theorem 3 ) such that

$$
\left\|f_{n}(t)-f_{n}(s)\right\| \leq M_{R_{0}} \cdot\left(g_{n}(t)-g_{n}(s)\right) \text { for every } 0 \leq s<t \leq 1 .
$$

Theorem 4 Let $G:[0,1] \times \mathbb{R}^{d} \rightarrow \mathcal{P}_{k}\left(\mathbb{R}^{d}\right)$ satisfy the assumptions of Theorem 3 , and let $\left(g_{n}\right)_{n}$ be an equiregulated sequence of left-continuous nondecreasing functions on $[0,1]$ with $g_{n}(0)=0$ satisfying the following condition:

$$
g_{n}(1)-g_{n}(0) \leq \frac{R_{0}-\left|G\left(0, x_{0}\right)\right|}{M_{R_{0}}}, \forall n .
$$

Then there exists a left-continuous nondecreasing function $g:[0,1] \rightarrow \mathbb{R}$ with

$$
g(1)-g(0) \leq \frac{R_{0}-\left|G\left(0, x_{0}\right)\right|}{M_{R_{0}}}
$$


such that on a subsequence, still denoted by $\left(g_{n}\right)_{n}$,

$$
g_{n} \rightarrow g \text { uniformly on }[0,1] \text {. }
$$

Moreover, if $\mathcal{S}_{g}$ is the set of solutions for the problem (1.1) driven by $\mu_{g}$ with related selections satisfying

$$
\|f(t)-f(s)\| \leq M_{R_{0}}(g(t)-g(s)) \quad \text { for every } 0 \leq s<t \leq 1,
$$

then for every sequence $\left(x_{n}\right)_{n}$, with $x_{n} \in \mathcal{S}_{g_{n}}$, there exists $x \in \mathcal{S}_{g}$ towards which a subsequence $\left(x_{n_{k}}\right)_{k}$ converges in the two-norm topology.

Proof The hypotheses of Theorem 3 are verified for $g_{n}$ for all $n \in \mathbb{N}$, therefore the sets $\mathcal{S}_{g_{n}}$ are nonempty.

The sequence $\left(g_{n}\right)_{n}$ is by hypothesis equiregulated, bounded in variation and moreover we have $g_{n}(0)=0$ for any $n$. Therefore by Lemma 1 there is a subsequence, still denoted by $\left(g_{n}\right)_{n}$ uniformly convergent to some $B V$ function $g$ whose total variation satisfies

$$
g(1)-g(0) \leq \frac{R_{0}-\left|G\left(0, x_{0}\right)\right|}{M_{R_{0}}} .
$$

It follows that $g$ is nondecreasing. Besides, the Moore-Osgood Theorem on exchanging limits ([41, page 140]) implies that (since all $g_{n}$ 's are left-continuous), $g$ is also left-continuous on $[0,1]$.

The hypotheses of Theorem 3 are satisfied also by $g$, thus the solutions set $\mathcal{S}$ is nonempty.

Let $\left(x_{n}\right)_{n}$ be a sequence of solutions for our problem driven by the measures $\mu_{g_{n}}$, respectively. For each $n$, there exists $f_{n}(t) \in G\left(t, x_{n}(t)\right)$ such that

$$
x_{n}(t)=x_{0}+\int_{[0, t)} f_{n}(s) d \mu_{g_{n}}(s), \forall t \in[0,1]
$$

and $f_{n}$ satisfies with $\left\|f_{n}(t)-f_{n}(s)\right\| \leq M_{R_{0}} \cdot\left(g_{n}(t)-g_{n}(s)\right)$ for any $s<t$.

The sequence $\left(f_{n}\right)_{n}$ is bounded in variation and $\left(f_{n}(0)\right)_{n} \subset G\left(0, x_{0}\right)$ which is compact.

Since $\left(g_{n}\right)_{n}$ is equiregulated, the sequence $\left(f_{n}\right)_{n}$ is equiregulated as well, thus, by reasoning in a similar way as in the proof of Theorem 3 , one can find a subsequence $\left(f_{n_{k}}\right)_{k}$ uniformly convergent to a $B V$ function $f:[0,1] \rightarrow \mathbb{R}^{d}$.

Define

$$
x(t)=x_{0}+\int_{[0, t)} f(s) d \mu_{g}(s), \text { for each } t \in[0,1] .
$$

By the upper semicontinuity assumption, it follows that $f(t) \in G(t, x(t))$ for all $t \in[0,1]$, therefore $x \in \mathcal{S}_{g}$, where $x$ is given by $x(t)=x_{0}+\int_{[0, t)} f(s) d \mu_{g}(s)$.

Let us now show that $\left(x_{n_{k}}\right)_{k}$ converges uniformly to $x$.

One can write

$$
\begin{aligned}
& \left\|x_{n_{k}}(t)-x(t)\right\|=\left\|\int_{[0, t)} f_{n_{k}}(s) d \mu_{g_{n_{k}}}(s)-\int_{[0, t)} f(s) d \mu_{g}(s)\right\| \\
& \leq \int_{[0, t)}\left\|\left(f_{n_{k}}-f\right)(s)\right\| d \mu_{g_{n_{k}}}(s)+\left\|\int_{[0, t)} f(s) d\left(\mu_{g_{n_{k}}}-\mu_{g}\right)(s)\right\| .
\end{aligned}
$$

The first term tends to 0 uniformly in $t \in[0,1]$ by Proposition 3.i) since $\left(f_{n_{k}}\right)_{k}$ tends uniformly to $f$ and for each $[a, b) \subset[0,1]$,

$$
g_{n}(b)-g_{n}(a) \leq \frac{R_{0}-\left|G\left(0, x_{0}\right)\right|}{M_{R_{0}}}, \forall n .
$$


The second term tends uniformly to 0 by Proposition 3.ii) because

$$
\int_{[0, t)} f(s) d\left(\mu_{g_{n_{k}}}-\mu_{g}\right)(s)=\int_{0}^{t} f(s) d\left(g_{n_{k}}-g\right)(s),
$$

$g_{n} \rightarrow g$ uniformly, $f$ is BV and $f(0) \in G\left(0, x_{0}\right)$.

So, the uniform convergence is verified.

Next, by a similar calculus as in the proof of Theorem 3 , the sequence $\left(x_{n}\right)_{n}$ is bounded in variation by

so

$$
R_{0} \cdot\left(g_{n}(1)-g_{n}(0)\right) \leq R_{0} \cdot \frac{R_{0}-\left|G\left(0, x_{0}\right)\right|}{M_{R_{0}}},
$$

$$
\left\|x_{n}\right\|_{B V} \leq\left\|x_{0}\right\|+R_{0} \cdot \frac{R_{0}-\left|G\left(0, x_{0}\right)\right|}{M_{R_{0}}}, \text { for every } n \in \mathbb{N} .
$$

In conclusion, $\left(x_{n_{k}}\right)_{k}$ converges to $x$ in the two-norm topology.

Remark 5 One can prove that for the sequence $\left(x_{n_{k}}\right)_{k}$ a result similar to [3, Theorem 1] holds: for each $h:[0,1] \rightarrow \mathbb{R}$ regulated,

$$
\int_{[0,1]} h(s) d x_{n_{k}}(s) \rightarrow \int_{[0,1]} h(s) d x(s) .
$$

Proof Let $h:[0,1] \rightarrow \mathbb{R}$ be a regulated function, by the Substitution [43, Theorem 2.3.19]:

$$
\begin{aligned}
& \left\|\int_{0}^{1} h(s) d x_{n_{k}}(s)-\int_{0}^{1} h(s) d x(s)\right\| \\
= & \left\|\int_{0}^{1} h(s) f_{n_{k}}(s) d g_{n_{k}}(s)-(K S) \int_{0}^{1} h(s) f(s) d g(s)\right\| \\
= & \left\|\int_{0}^{1} h(s)\left(f_{n_{k}}-f\right)(s) d g_{n_{k}}(s)+\int_{0}^{1} h(s) f(s) d\left(g_{n_{k}}(s)-g(s)\right)\right\| \\
\leq & \left\|\int_{0}^{1} h(s)\left(f_{n_{k}}-f\right)(s) d g_{n_{k}}(s)\right\|+\left\|\int_{0}^{1} h(s) f(s) d\left(g_{n_{k}}(s)-g(s)\right)\right\| \\
\leq & \int_{0}^{1}|h(s)|\left\|f_{n_{k}}(s)-f(s)\right\| d g_{n_{k}}(s)+\left\|\int_{0}^{1} h(s) f(s) d\left(g_{n_{k}}(s)-g(s)\right)\right\|
\end{aligned}
$$

which is arbitrarily small when $k \rightarrow \infty$. Indeed, the first term is small because $h$ is bounded, in the second term this is a consequence of [3, Theorem 1] since the product of two regulated functions is regulated.

\subsection{Minimality Result}

Consider $G:[0,1] \times \mathbb{R}^{d} \rightarrow \mathcal{P}_{k}\left(\mathbb{R}^{d}\right)$ a compact-valued multifunction satisfying hypotheses 1), 2), 3) of Theorem 3.

We aim to minimize the functional

$$
\int_{0}^{1} k\left(t, x(t), \mu_{g}\right) d t+l(x)
$$


over a set of pairs $\left(\mu_{g}, x\right)$, where $\mu_{g}$ is a measure generated by some left-continuous nondecreasing function $g:[0,1] \rightarrow \mathbb{R}$ for which the differential inclusion

$$
\begin{aligned}
& d x(t) \in G(t, x(t)) d \mu_{g}(t), \\
& x(0)=x_{0}
\end{aligned}
$$

driven by the measure $\mu_{g}$ has solutions and $x:[0,1] \rightarrow \mathbb{R}^{d}$ is a solution.

Denote by $\mathcal{M}$ the set of of measures generated by left-continuous nondecreasing functions which are null at 0 , satisfying the condition (3.3), thus ensuring the existence of solutions for (1.1).

For the convenience of the reader, let us recall that:

Definition 5 A function $h: Y \rightarrow \mathbb{R} \cup\{ \pm \infty\}$ is called:

i) sequentially lower semicontinuous at a point $y_{0} \in Y$ if for every sequence $y_{n}$ converging to $y_{0}, h\left(y_{0}\right) \leq \lim \inf h\left(y_{n}\right)$; sequentially lower semicontinuous if it is sequentially lower semicontinuous at any point $y_{0}$;

ii) sequentially coercive if for every $\lambda \in \mathbb{R}$, the set $L_{\lambda}=\overline{\{y \in Y: h(y) \leq \lambda\}}$ is sequentially compact in $Y$.

We shall make use of the following result asserting the existence of a minimum:

Theorem 5 ([16, Theorem 1.3.19]) Let $Y$ be a topological space and $h: Y \rightarrow \mathbb{R} \cup\{ \pm \infty\}$ be sequentially coercive and sequentially lower semicontinuous. Then $h$ has a minimum in $Y$.

Now suppose that:

H1) the function $k:[0,1] \times \mathbb{R}^{d} \times \mathcal{M} \rightarrow \mathbb{R}_{+}$is sequentially lower semicontinuous w.r.t. the last two arguments when $\mathcal{M}$ is endowed with the weak* topology and

$\mathrm{H} 2)$ the map $l: B V\left([0,1], \mathbb{R}^{d}\right) \rightarrow \mathbb{R}$ is sequentially lower semicontinuous w.r.t. the two-norm topology.

The main result of this section, on minimality conditions related to measure differential inclusions, is presented below.

Theorem 6 Let $\mathcal{G}$ be a subset of $\mathcal{M}$ generated by an equiregulated family of functions. Let $G:[0,1] \times \mathbb{R}^{d} \rightarrow \mathcal{P}_{k}\left(\mathbb{R}^{d}\right)$ satisfy hypotheses of Theorem $3, k:[0,1] \times \mathbb{R}^{d} \times \mathcal{G} \rightarrow \mathbb{R}_{+}$ have property $H 1)$ and $l: B V\left([0,1], \mathbb{R}^{d}\right) \rightarrow \mathbb{R}$ verify $\left.H 2\right)$.

Then the set

$$
\left\{\int_{0}^{1} k\left(t, x(t), \mu_{g}\right) d t+l(x): \mu_{g} \in \mathcal{G}, x \in \mathcal{S}_{g}\right\}
$$

has a minimum.

Proof Let us show that the functional $h$ defined on $\left\{\left(x, \mu_{g}\right): g \in \mathcal{G}, x \in \mathcal{S}_{g}\right\} \subset$ $B V\left([0,1], \mathbb{R}^{d}\right) \times \mathcal{G}$ by

$$
h\left(x, \mu_{g}\right)=\int_{0}^{1} k\left(t, x(t), \mu_{g}\right) d t+l(x)
$$

satisfies the hypotheses of Theorem 5 . 
The sequential lower semicontinuity follows from H1) and H2). Indeed, let $\mu_{g_{n}} \rightarrow \mu_{g}$ in $\mathcal{G}$ and a sequence of associated solutions $x_{n} \rightarrow x$ in the two-norm topology. Then $x_{n}(t) \rightarrow$ $x(t)$ for each $t \in[0,1]$, so

$$
k\left(t, x(t), \mu_{g}\right) \leq \liminf k\left(t, x_{n}(t), \mu_{g_{n}}\right), \text { for every } t \in[0,1]
$$

whence

$$
\int_{0}^{1} k\left(t, x(t), \mu_{g}\right) d t \leq \int_{0}^{1} \liminf k\left(t, x_{n}(t), \mu_{g_{n}}\right) d t .
$$

By Fatou Lemma one gets

$$
\int_{0}^{1} k\left(t, x(t), \mu_{g}\right) d t \leq \liminf \int_{0}^{1} k\left(t, x_{n}(t), \mu_{g_{n}}\right) d t
$$

and so, using also hypothesis H2),

$$
\begin{aligned}
h\left(x, \mu_{g}\right) & =\int_{0}^{1} k\left(t, x(t), \mu_{g}\right) d t+l(x) \\
& \leq \liminf \int_{0}^{1} k\left(t, x_{n}(t), \mu_{g_{n}}\right) d t+\liminf l\left(x_{n}\right) \\
& \leq \liminf \left(\int_{0}^{1} k\left(t, x_{n}(t), \mu_{g_{n}}\right) d t+l\left(x_{n}\right)\right) \\
& =\liminf h\left(x_{n}, \mu_{g_{n}}\right) .
\end{aligned}
$$

In order to prove the sequential coercivity, consider $\lambda \in \mathbb{R}$ and a sequence $\left(x_{n}, \mu_{g_{n}}\right)_{n} \subset$ $\left\{\left(x, \mu_{g}\right) \in \mathcal{S}_{g} \times \mathcal{G}: h\left(x, \mu_{g}\right) \leq \lambda\right\}$. Then

$$
\int_{0}^{1} k\left(t, x_{n}(t), \mu_{g_{n}}\right) d t+l\left(x_{n}\right) \leq \lambda, \quad \forall n \in \mathbb{N} .
$$

By hypothesis,

$$
g_{n}(1)-g_{n}(0) \leq \frac{R_{0}-\left|G\left(0, x_{0}\right)\right|}{M_{R_{0}}}, \quad \forall n \in \mathbb{N} .
$$

The sequence $\left(g_{n}\right)_{n}$ is equiregulated and $g_{n}(0)=0$ for each $n$, therefore by Lemma 1 it follows that there exists a subsequence $\left(g_{n_{k}}\right)_{k}$ uniformly convergent to a $B V$ function $g:[0,1] \rightarrow \mathbb{R}$. Moreover $g$ is left-continuous and nondecreasing and

$$
g(1)-g(0) \leq \frac{R_{0}-\left|G\left(0, x_{0}\right)\right|}{M_{R_{0}}} .
$$

Note that [3, Theorem 1] implies that $\left(\mu_{g_{n_{k}}}\right)_{k}$ weakly* converges to $\mu_{g}$ as $k \rightarrow \infty$.

Now we use Theorem 4 and from the sequence of solutions $x_{n_{k}}$ we can extract a subsequence, not relabelled, two-norm convergent to a solution $x$ of (1.1) driven by $\mu_{g}$. 
We need to prove that $\int_{0}^{1} k\left(t, x(t), \mu_{g}\right) d t+l(x) \leq \lambda$. Again, this follows from the hypotheses since $\left(x_{n_{k}}\right)_{k}$ two-norm converges to $x$ in $B V\left([0,1], \mathbb{R}^{d}\right)$ and $\left(\mu_{g_{n_{k}}}\right)_{k}$ weakly* converges to $\mu_{g}$ in $\mathcal{G}$ :

$$
\begin{aligned}
\int_{0}^{1} k\left(t, x(t), \mu_{g}\right) d t+l(x) & \leq \int_{0}^{1} \liminf k\left(t, x_{n_{k}}(t), \mu_{g_{n_{k}}}\right) d t+\liminf l\left(x_{n_{k}}\right) \\
& \leq \liminf \int_{0}^{1} k\left(t, x_{n_{k}}(t), \mu_{g_{n_{k}}}\right) d t+\liminf l\left(x_{n_{k}}\right) \\
& \leq \liminf \left(\int_{0}^{1} k\left(t, x_{n_{k}}(t), \mu_{g_{n_{k}}}\right) d t+l\left(x_{n_{k}}\right)\right) \leq \lambda
\end{aligned}
$$

and so, $\left(x, \mu_{g}\right) \in\left\{\left(x, \mu_{g}\right) \in B V\left([0,1], \mathbb{R}^{d}\right) \times \mathcal{G}: h\left(x, \mu_{g}\right) \leq \lambda\right\}$ and the sequential coercitivity is verified.

Remark 6 In fact, from [3, Theorem 1] it is obvious that we could have required only that the function $k$ is lower semicontinuous w.r.t. the last two arguments when $\mathcal{M}$ is endowed with the following topology: $\mu_{n} \rightarrow \mu$ if and only if for each regulated function $f:[0,1] \rightarrow \mathbb{R}$,

$$
\int_{[0,1]} f(s) d \mu_{n}(s) \rightarrow \int_{[0,1]} f(s) d \mu(s) .
$$

Remark 7 Finally, we notice that we are aware of the fact that Theorem 6 provides the existence of a solution to the minimization problem over a subset of measures and a specific subset of associated solutions to the measure differential inclusion (1.1), while in $[29,31,32]$ or [28] the minimal pair (control, state) is obtained over all measures and all associated solutions of differential problem. However, we would like to stress that our setting is much different: we work in the wider case of multivalued functions and $G$ satisfies conditions involving only the bounded variation w.r.t. the Pompeiu excess and not the Pompeiu-Hausdorff distance (in contrast to [29, 31, 32], where it is a single-valued function continuous, uniformly Lipschitz w.r.t. $x$, having linear growth or to [28] where continuity in all arguments and local Lipschitz assumptions are imposed).

Therefore, it provides a new method to solve a widely encountered minimization problem ([44]) in situations where previous results do not apply. For instance, even in the single-valued case, for non-Lipschitz function $G$ we are able to find solutions (while the theory in [31] does not work). Also, in the set-valued setting, in addition to [28], we cover the case where $G$ is not necessarily continuous with respect to all arguments and locally Lipschitz.

Acknowledgements The authors are greatly indebted to the associate editor and to the two referees for their insightful comments and constructive suggestions.

This research has been partially supported by GNAMPA, prot. U-UFMBAZ-2018-000351. The third author has been supported by GNAMPA, prot. U-UFMBAZ-2018-001501 and by "Excellence in Advanced Research, Leadership innovation and Patenting for University and Regional Development" - EXCALIBUR, Grant Contract no. 18PFE / 10.16.2018 Institutional Development Project - Funding for Excellence in RDI, Program 1 - Development of the National R \& D System, Subprogram 1.2 - Institutional Performance, National Plan for Research and Development and Innovation for the period 2015-2020 (PNCDI III).

Funding Open access funding provided by Università degli Studi di Palermo within the CRUI-CARE Agreement. 
Open Access This article is licensed under a Creative Commons Attribution 4.0 International License, which permits use, sharing, adaptation, distribution and reproduction in any medium or format, as long as you give appropriate credit to the original author(s) and the source, provide a link to the Creative Commons licence, and indicate if changes were made. The images or other third party material in this article are included in the article's Creative Commons licence, unless indicated otherwise in a credit line to the material. If material is not included in the article's Creative Commons licence and your intended use is not permitted by statutory regulation or exceeds the permitted use, you will need to obtain permission directly from the copyright holder. To view a copy of this licence, visit http://creativecommonshorg/licenses/by/4.0/.

\section{References}

1. Soledad Aronna, M., Rampazzo, F.: $L^{1}$ limit solutions for control systems. J. Differential Equations 258, 954-979 (2015)

2. Aubin, J.-P., Frankowska, H.: Set-valued Analysis. Birkhäuser, Boston (1990)

3. Aye, K.K., Lee, P.Y.: The dual of the space of bounded variation. Math. Bohem. 131(1), 1-9 (2006)

4. Belov, S.A., Chistyakov, V.V.: A selection principle for mappings of bounded variation. J. Math. Anal. Appl. 249, 351-366 (2000)

5. Billingsley, P.: Weak convergence of measures: Applications in probability. In: CBMS-NSF Regional Conference Series in Applied Mathematics (1971)

6. Boccuto, A., Candeloro, D., Sambucini, A.R.: Stieltjes-type integrals for metric semigroup-valued functions defined on unbounded intervals. PanAm. Math. J. 17(4), 39-58 (2007)

7. Çamlibel, M.K., Heemels, W.P.M.H., van der Schaft, A.J., Schumacher, J.M.: On the existence and uniqueness of solution trajectories to hybrid dynamical systems. In: Nonlinear and Hybrid Systems in Automotive Applications, pp. 391-422 (2002)

8. Castaing, C., Valadier, M.: Convex Analysis and Measurable Multifunctions Lecture Notes in Math, vol. 580. Springer, Berlin (1977)

9. Cichoń, M., Satco, B.: Measure differential inclusions - between continuous and discrete. Adv. Diff. Equations 56, 18 (2014). https://doi.org/10.1186/1687-1847-2014-56

10. Cichoń, M., Satco, B.: On the properties of solutions set for measure driven differential inclusions. Discrete and continuous dynamical systems. Special Issue: SI, pp. 287-296 (2015)

11. Cichon, M., Satco, B., Sikorska-Nowak, A.: Impulsive nonlocal differential equations through differential equations on time scales. Appl. Math. Comp. 218, 2449-245 (2011)

12. Chistyakov, V.V.: Asymmetric variations of multifunctions with application to functional inclusions. J. Math. Anal. Appl. 478(2), 421-444 (2019)

13. Chistyakov, V.V., Repovš, D.: Selections of bounded variation under the excess restrictions. J. Math. Anal. Appl. 331, 873-885 (2007)

14. Code, W.J., Loewen, P.D.: Optimal control of non-convex measure-driven differential inclusion. SetValued Anal. 19, 203-235 (2010)

15. Dal Maso, G., Rampazzo, F.: On System of ordinary differential equations with measures as controls. Differ. Integral Equ. 4(4), 739-765 (1991)

16. Denkowski, Z., Migorski, S., Papageorgiou, N.S.: An introduction to nonlinear analysis: Theory. Kluwer Academic Publishers (2003)

17. Di Piazza, L., Marraffa, V., Satco, B.: Closure properties for integral problems driven by regulated functions via convergence results. J. Math. Anal. Appl. 466, 690-710 (2018)

18. Di Piazza, L., Marraffa, V., Satco, B.: Approximating the solutions of differential inclusions driven by measures. Ann. Mat. Pura Appl. 198, 2123-2140 (2019)

19. Federson, M., Mesquita, J.G., Slavík, A.: Measure functional differential equations and functional dynamic equations on time scales. J. Diff. Equations 252, 3816-3847 (2012)

20. Fraňková, D.: Regulated functions. Math. Bohem. 116(1), 20-59 (1991)

21. Frigon, M., López Pouso, R.: Theory and applications of first-order systems of Stieltjes differential equations. Adv. Nonlinear Anal. 6, 13-36 (2017)

22. Goebel, R., Teel, A.R.: Solutions to hybrid inclusions via set and graphical convergence with stability theory applications. Automatica 42, 573-587 (2006)

23. Karamzin, D.Y., De Oliveira, V.A., Pereira, F.L., Silva, G.N.: On the properness of an impulsive control extension of dynamic optimization problems. ESAIM: COCV 21, 857-875 (2015)

24. Kurzweil, J.: Generalized ordinary differential equations and continuous dependence on a parameter. Czechoslovak Math. J. 7(82), 418-449 (1957) 
25. Miller, B., Rubinovitch, E.Y.: Impulsive Control in Continuous and Discrete-Continuous Systems. Kluwer Academic Publishers, Dordrecht (2003)

26. Monteiro, G.A., Slavik, A.: Extremal solutions of measure differential equations. J. Math. Anal. Appl. 444, 568-597 (2016)

27. Monteiro, G.A., Slavik, A., Tvrdy, M.: Kurzweil-Stieltjes integral. Theory and its Applications, World Scientific Series in real analysis. Vol. 15 (2018)

28. Motta, M., Sartori, C.: On $l^{1}$ limit solutions in impulsive control. DCDIS Series S 11(6), 1201-1218 (2018)

29. Murray, J.M.: Existence theorems for optimal control and calculus of variations problems where the states can jump. Siam J. Control Optim. 24, 412-438 (1986)

30. Phillips, G.M.: Interpolation and Approximation by Polynomials. Springer, Berlin (2003)

31. Raymond, J.P.: Optimal control problems in spaces of functions of bounded variation. Differential Integral Equations 10(1), 105-136 (1997)

32. Raymond, J.P., Seghir, D.: Existence and characterization of BV-curves for problems of calculus of variations. Nonlinear Anal. T.M.A. 28, 109-1132 (1997)

33. Saks, S.: Theory of the Integral. Monografie Matematyczne, Warszawa (1937)

34. Satco, B.: Continuous dependence results for set-valued measure differential problems. Electr. Jour. Qualit. Th. Diff. Equat. 79, 1-15 (2015)

35. Satco, B.: Nonlinear Volterra integral equations in Henstock integrability setting. Electr. J. Diff. Equ., 39 (2008)

36. Schwabik, Š.: Generalized ordinary differential equations. World Scientific (1992)

37. Schwabik, Š., Tvrdý, M., Vejvoda, O.: Differential and Integral Equations. Boundary Problems and Adjoints, Dordrecht, Praha (1979)

38. Silva, G.N., Vinter, R.B.: Measure driven differential inclusions. J. Math. Anal. Appl. 202, 727-746 (1996)

39. Silva, G.N., Vinter, R.B.: Necessary conditions for optimal impulsive control problems. SIAM J. Control Optim. 35(6), 1829-1846 (1997)

40. Slavík, A.: Well-posedness results for abstract generalized differential equations and measure functional differential equations. J. Differential Equations 259, 666-707 (2015)

41. Taylor, A.E.: General Theory of Functions and Integration. Dover Books on Mathematics Series (2012)

42. Toth, G.: Measures of Symmetry for Convex Sets and Stability. Springer, New York (2015)

43. Tvrdý, M.: Differential and Integral Equations in the Space of Regulated Functions. Habil, Thesis, Praha (2001)

44. Vinter, R.: Optimal control. Birkhäuser (2000)

45. Wiweger, A.: Linear spaces with mixed topology. Studia Math. 20(1), 47-68 (1961)

46. Zavalishchin, S.T., Sesekin, A.N.: Dynamic Impulse Systems. Kluwer Academic, Dordrecht (1997)

Publisher's Note Springer Nature remains neutral with regard to jurisdictional claims in published maps and institutional affiliations. 